\title{
Research Square \\ Performance Analysis of Linear Precoding in Downlink Based on Polynomial Expansion on massive MIMO systems
}

SAMMAIAH THURPATI ( $\square$ sammaiah_404@yahoo.com )

National Institute of Technology Tiruchirappalli

$P$ Muthuchidambaranathan

NIT-Trichy: National Institute of Technology Tiruchirappalli

\section{Research Article}

Keywords: massive MIMO, precoding, zero forcing, matched filter, truncated polynomial expansion, regularized zero-forcing, average achievable rate

Posted Date: September 21st, 2021

DOI: https://doi.org/10.21203/rs.3.rs-787294/v1

License: (c) (i) This work is licensed under a Creative Commons Attribution 4.0 International License.

Read Full License

Version of Record: A version of this preprint was published at Journal of Physics: Conference Series on November 1st, 2021. See the published version at https://doi.org/10.1088/1742-6596/2062/1/012006. 


\title{
Performance Analysis of Linear Precoding in Downlink Based on Polynomial Expansion on massive MIMO systems
}

\author{
Sammaiah Thurpati ${ }^{1}$, \\ Muthuchidambaranathan $\mathbf{p}^{2}$ \\ National Instittute of \\ Technology ${ }^{1,2}$, Tiruchirappalli \\ 9 Received: DD Month YEAR / Accepted: DD Month YEAR
}

Abstract The performance of linear precoding schemes in downlink Massive MIMO systems is dealt with in this paper. Linear precoding schemes are incorporated with maximum ratio transmission (MRT) and zero forcing (ZF) , truncated polynomial expansion (TPE), regularized zero force (RZF) in Downlink massive MIMO systems. Massive MIMO downlink output is evaluated with linear precoding included. This paper expresses the performance of achievable sum rate linear precoding with variable signal-to-noise (SNR) ratio and achievable sum rate and several transmitter-receiver antennas, such as imperfect CSI, fewer complex processing and inter-user interference. The transmitter has complete state information on the channel. The information narrate how a signal propagates to the receiver from the transmitter and reflects, for example, fading cumulative effect of distance scattering and power decay. They show that the performance analysis of two linear precoding techniques,i.e.Maximum Ratio Transmission (MRT) and Zero Forcing (ZF) for downlink mMIMO output network over a perfect chain. The results show the improved $\mathrm{ZF}$ precoding achievable sum rate compared to the MRT precoding schemes and also compared the average achievable rate RZF and TPE.
\end{abstract}

Keywords massive MIMO, precoding, zero forcing, matched filter, truncated polynomial expansion, regularized zero-forcing, average achievable rate.

\section{Introduction}

Nowadays, the mobile communication network having several users is growing 31 exponentially. Through mobile communications technology, users must have a 32 more data rate low latency and full mobility communications networks. Mobile 3 communication technology needs to upgrade the infrastructure to satisfy the

Address(es) of author(s) should be given 
demands of the market. To reach a broader bandwidth, the mobile communications network is now moving into the 5th generation and working on the massive MIMO and millimeter wave spectrum[1].

However, the 5th generation organizes the recently developed subsystems on its network, one is an antenna subsystem and the other one is a beamforming subsystems. A multi-input multi-output (MIMO) antenna subsystems is implemented by the latest mobile communications technology. When the massive MIMO there is a large number of in antennas. Therefore one is a transmitter and the other one is a receiver. The massive MIMO has certain leads in terms of channel power, spectral efficiency, reducing interference[1]. Because of its ability to bit error rate, signal-to-noise ratio, feasible sum rate and reduce interference, the mMIMO was a tropical research matter. The transmitter is fitted with large numbers of antennas assisting multiple users with single or multiplied receiver antennas. There will be multipaths between transmitter and receiver, multiple users are served simultaneously. Interference under these conditions may theoretically occur.

mMIMO technology has been also recently called the huge Scale MIMO. This mMIMO technology is a huge base station (BS) transmitting antennas $[6]$ and a small number of offering antennas are used by the user terminal(UE) for communication systems. The major interference of the suppression gain and the array gain from the mMIMO that allows the control consumer spectral efficiency and the cell total spectral efficiency have been significantly improved[2].mMIMO technology is now attracting considerable academic and industrial attention. Most studies were considering the performance of uplinks. In this paper, we are researching a huge downlink framework from mMIMO with linear precoding schemes.

The mMIMO provides spectral efficiency increases in energy efficiency and radiation compared with the $4 \mathrm{G}$ wireless technologies. Hence, the massive MIMO technology is a forward-looking innovative $5 \mathrm{G}$ communication technology[2-3]. The high consumption of downlink overhead channels and feedback required to obtain the CSI of each user will eventually limit the number of BS antennas[4]. BS downlink transmission can effectively reduce the associated signaling overhead according to the uplink channel estimate.

Precoding is a part of the massive processing of MIMO signals. A precoding/beamforming module introduced would add massive enhancements to MIMO. Precoding is made up of two forms, one of which is non-linear and the other linear. Several earlier studies have expressed the efficiency of both of them. The authors in[3] proposed zero-forcing precoding efficiency and studied the relationship for precoding maximum ratio transmission between vector and matrix normalization.

Meanwhile, in the sense of MIMO's huge downlink in[4 ], the author has studied the feasibility of maximum ratio transmission and zero-forcing. $\operatorname{In}[5]$ the authors discussed the success in downlink massive MIMO for non-linear precoding of the combination of statistical and imperfect channel state information (CSI).This concept explores the precoding techniques of zero-forcing 
and maximum transmission ratio as well as the regularized zero-forcing (RZF), truncated polynomial expansion (TPE) in downlink mMIMO.

This paper studies the linear precoding in downlink massive MIMO with a channel state information transmitter (CSIT). Linear precoding involves maximum transmission ratio (MRT) and zero-forcing (ZF), regularized zero-forcing (RZF), truncated polynomial expansion (TPE). The paper is structured according to the following. Section I presents the introduction, previous plays, and the history of this study. The model of this work for the mMIMO system is outlined in section II. Section III will present the results and explanation of the pprecoding performance Section IV will conclude this study.

\section{System Model}

The downlink of a mMIMO scheme is treated as a single cell in this study, in which a BS with $\mathrm{M}$ antennas transmits data to $\mathrm{K}$ user terminals via a single antenna (UTs). The channel that has a Rayleigh fading channel with zero mean and a variance of $\lambda_{k}$ (Fig. 1). This work employs a single-cell model. Fig 1 represents a mmimo model of the single-cell downlink. For all users, the transmitter has perfect CSI. The Massive MIMO platform uses configuration Rayleigh channel[18]. The channel which has the transmitter and user coupling is shown in Fig.1 Furthermore, the precoding locality is also viewed in Fig.2.As illustrated in Fig.1,mMIMO device consisting of the BS equipped with M antennas and UTs, each UT provided with one antenna, We assume in this paper that the BS supporting UTs over the Rayleigh fading channel will obtain perfect channel state information on a certain frequency or subcarrier

The signal vector transmitted to the $\mathrm{K}$ users during the downlink transmission, where $\mathrm{M} i \mathrm{~K}$ can be expressed as

$$
r=\sqrt{\rho} W_{S}
$$

where $W \in C^{M \times K}$ is the linear precoding matrix , $S \in C^{M \times 1}$ is the precoding source data, and $\rho$ is the moderate transmission power of the BS. Here, both $\mathrm{M}$ and $\mathrm{K}$ are huge and their ratio is suppose to be persistent [24]. The precoding matrix $\mathrm{W}$ is a jusification of the channel matrix defines by $H \in C^{M \times K}$ The power of the origin signal being transmit is normalized, i.e. $s^{2}=1$

Let ' $\mathrm{r}$ ' is $M \times 1$ Precoded vector with a complex information symbol transmitted from the base station antenna.The signal that the $y \in C^{K \times 1}$ user antenna receives as [ 13 ]

$$
\begin{gathered}
y=H^{H} r+n_{s} \\
r=\sqrt{\rho} H^{H} W_{S}+n_{s}
\end{gathered}
$$

where $\mathrm{H}$ is the $M \times K$ channel matrix in the middle of the $\mathrm{M}$ is an antenna at $\mathrm{BS}$ and the $\mathrm{K}$ is device terminals. $n_{s} \in C^{K \times 1}$ is the additive white gaussian noise (AWGN) with zero mean and variance $\sigma^{2}=N_{0} B$. Here the AWGN channel length and spectral power density denote by B $(\mathrm{Hz})$ and $N_{0}(\mathrm{~W} / \mathrm{Hz})$ respectively 


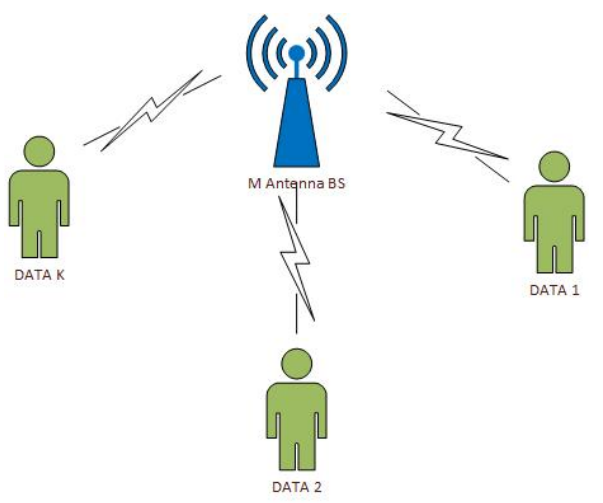

Fig. 1 massive mimo system on downlink

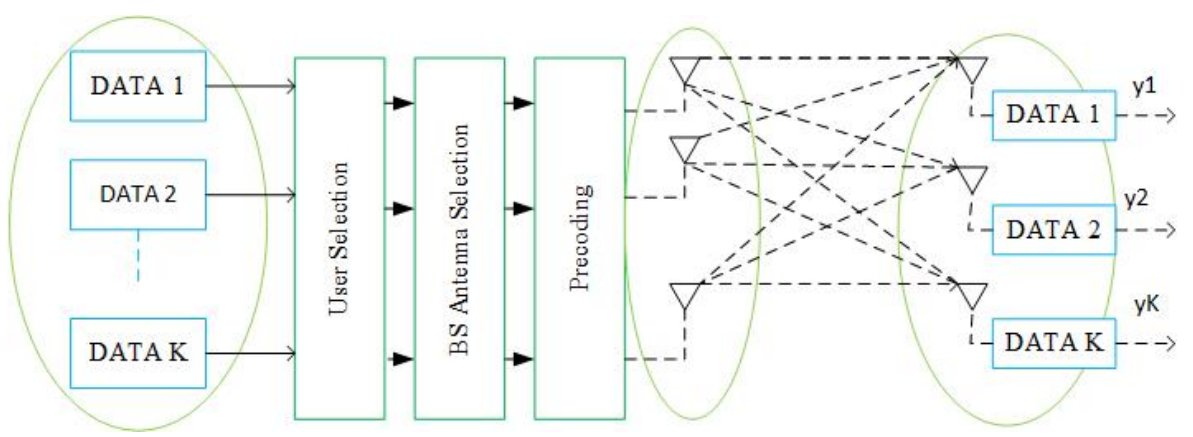

Fig. 2 linear precoding system in massive mimo

MRT and ZF precoding has often been introduced for mMIMO signal processing due to good quality and ease of operation. The ZF and MRT precoding weight may be work out as follows[10][11 ], respectively.

$$
A_{Z F}=H^{H}\left(H H^{H}\right)^{-1}
$$

$$
A_{M R T}=H^{H}
$$

For $\mathrm{K}$ and a huge number of $\mathrm{Z}$, the $k_{t h}$ user interference signal to noise ratio, $\mathrm{ZF}$ and MRT precoding are prepared as follows.

$$
S I N R_{K}^{Z F}=\frac{p_{d}(Z-K)}{K}
$$

124

$$
S I N R_{K}^{M R T}=\frac{p_{d}(Z)}{K\left(p_{d}+1\right)}
$$




\subsection{Normalization method}

The linear precoder with zero-forcing and maximum transmission is known as the normalization of the vector and the matrix. The normalized transmission of vectors or matrix beamforming vectors are given as $a_{k}=a_{1} /\left(\sqrt{K}\left\|a_{k}\right\|\right)$ and $a_{k}=a_{k} /\|A\|$ respectively, The normalization of the vector applies equal power per downlink channel, whereas the matrix normalization relents various power streams.

\subsection{1 vector normalization of $Z F / M R T$}

The received signal at the $k_{t h} \mathrm{UE}$ can be expressed in equation below

$$
y_{t}=\sqrt{p_{d}} h_{k}^{T} \frac{a_{k}}{\sqrt{K}|| a_{k} \|} s_{k}+\sqrt{p_{d}} \sum_{l=1, l \neq 1}^{k} h_{k}^{T} \frac{a_{1}}{\sqrt{K}|| a_{1} \|} s_{k}+n_{k}
$$

\subsection{2 matrix normalization of $Z F / M R T$}

The received signal can be write matrix normalization as in equation below

$$
y_{t}=\sqrt{p_{d}} h_{k}^{T} \frac{a_{k}}{\left\|a_{k}\right\|} s_{k}+\sqrt{p_{d}} \sum_{l=1, l \neq 1}^{k} h_{k}^{T} \frac{a_{1}}{\left\|a_{1}\right\|} s_{k}+n_{k}
$$

\section{Achievable sum Rate}

The achievable sum rate of precoders ZF and MRT is argued in [12 ], Assuming full downlink power is set and splited uniformly all users. The Shannon theorem acquires the achievable rate over the additive white Gaussian noise (AWGN) as a factor of the signal-to-noise ratio $(\mathrm{SNR})[12]$.

$$
D=\log _{2}(1+S N R)
$$

The SNR and CSI is a very crucial matter in multiuser communication systems. Normally each user emits data streams of multiple transmitters periodically and systematically to the CSI[11 ]. All of the transmitters receives the channel evaluation response from the receiver to the reverse-path so the transmitter acquires CSI. The transmitter, therefore, communicate with only the complete CSI with all receivers[9 ]. As can be seen in equation (2), additive noise and interference between the users itself is the signal emitted to each unit. Therefore, in a single cell downlink mMIMO network the obtainable information rate per user is defined with perfect channel state information.

$$
D_{k}=\log _{2}\left(1+S I N R_{k}\right)
$$

Where SINR of the $k^{t h}$ user is $S I N R_{k}$. The achieveable sum rate of ZF and MF precoders with optimal CSI for huge values of M and K [5 ]

The achievable sum rate of $\mathrm{K}$ users as formulated as :

$$
D_{\text {sum }}=K \log _{2}\left(1+S I N R_{k}\right)
$$




\section{Achievable sum Rate with ZF}

Formula (12) has been applied in zero forcing, the following are described[7] [8].

$$
D_{\text {sum }}^{Z F}=K \log _{2}\left(1+S I N R_{k}^{Z F}\right)
$$

Substituting (6) into (13), gives

$$
D_{\text {sum }}^{Z F}=K \log _{2}\left[1+\frac{p_{d}(z-K)}{K}\right]
$$

The zero forcing using vector normalization / matrix normalization methods is given in the following equation

$$
D_{Z F_{\text {vec }}}=D_{Z F_{\text {mat }}}=K \log _{2}\left[1+\frac{p_{d}(Z-K)}{K}\right]
$$

\section{Achievable sum Rate with MRT}

The MRT of the achievable sum rate is also deductible from (12) as

$$
D_{\text {sum }}^{M R T}=K \log _{2}\left(1+S I N R_{k}^{M R T}\right)
$$

Substituting (7) into (16), gives

$$
D_{\text {sum }}^{M R T}=K \log _{2}\left[1+\frac{p_{d}(Z)}{K\left(p_{d}+1\right)}\right]
$$

MRT is using methods of vector normalization / matrix normalization is given by below

$$
D_{M R T_{\text {vec }}}=D_{M R T_{m a t}}=K \log _{2}\left[1+\frac{p_{d}(Z)}{K\left(p_{d}+1\right)}\right]
$$

As the number of transmitting antennas hike with $\mathrm{Z} i ., \mathrm{K}$, Equations (14) and (17) indicate that the same downlink transmit power available and a rigid number of mobile users. ZF reaches data rates higher than MRT.

\section{Regularized Zero Forcing Precoding in mMIMO Systems}

The BS is armed with M antennas and supports K User Terminal with single antenna. The complex baseband signal obtained represents the collection of complex numbers per $\mathrm{C}$

$$
y_{k}=h_{k}^{H} X+n_{k} k=1, \ldots, K
$$

where $X \in C^{M \times 1}$ is transmited signal and $h_{k}$ Represents a specific variable to the BS channels and the $k^{\text {th }} \mathrm{UT}$. The spatially linear additive Gaussian 
noise $k^{t h} \mathrm{UT}$ is expressed by the $n_{k} \sim C N\left(0, \sigma^{2}\right)$ for $\mathrm{k}=1, \ldots, \mathrm{K}$, where $\sigma^{2}$ is variance of the noise

The BS utilizes Gaussian code and precoding,In light of this suspicion, the transmited signal in (19) can be communicated as

$$
X=\sum_{n=1}^{K} u_{n} v_{n}=U v
$$

The matrix representation is defined by charter $U=\left[u_{1} \ldots . u_{K}\right] \in C^{M \times K}$ be matrix of the precoding and $v=\left[v_{1} \ldots v_{K}\right]^{T} \sim C N\left(0_{K \times 1}, I_{K}\right)$ is the vector carries all symbols of UT data.

So,the signal received (19) can be indicates as

$$
y_{k}=h_{k}^{H} u_{k} v_{k}+\sum_{n=1, n \neq k}^{K} h_{k}^{H} u_{n} v_{n}+n_{k}
$$

The signal-to-interferencenoise ratio (SINR) at the $k^{\text {th }}$ UT [17] changes

$$
S I N R=\frac{h_{k}^{H} u_{k} u_{k}^{H} h_{k}}{h_{k}^{H} U_{k} U_{k}^{H} h_{k}+\sigma^{2}}
$$

By taking that each perfect insantaneous CSI has UT, the achievable rates in the UTs are

$$
r_{k} \simeq \log _{2}\left(1+S I N R_{k}\right), k=1, \ldots ., K
$$

Regularized zero-forcing (RZF) precoder was known as a linear precoder for mMIMO wirless communication systems due to its ability to trade compensation for MRT and ZF precoders[13],[14],[15],[16 ].

assume the total power constraints

$$
\frac{1}{K} \operatorname{tr}\left(U U^{H}\right)=P
$$

we specify the scaling factor $\frac{1}{K}$ counteracts the channel variance scaling, and $\operatorname{tr}($.$) is the trace function. We density the total power \mathrm{P}$ is set, Though we allow antennas number $\mathrm{K}$ and UTs M grow large.

Like that to [22], we specify the ZRF precoding as

$$
U_{Z R F}=\gamma\left(\widehat{H}^{H} \widehat{H}+\zeta I_{k}\right)^{-1}=\gamma \widehat{H}\left(\widehat{H} \widehat{H}^{H}+\zeta I_{M}\right)^{-1} \widehat{H}
$$

Where the variable for power normalization $\gamma$ Is set so that $U_{Z R F}$ achieves the power limit in (21). Regularization of the scalar coefficient. The $\zeta$ can be chosen the various ways, based on $\mathrm{P}, \sigma^{2}, \tau$, and device proportion.

The user efficiency characteristic in $S I N R_{k}$ in(22). Whereas the SINR is a randomized quantity. The SINR be conditional on the instant random user channel values in $\mathrm{H}$ and the instant estimate of $\widehat{H}$, the large (M, K) regime[23-26 ] can be used to estimate deterministic quantities. Such tests change based on channel statistics and are frequently mentioned to as finding equivalents, as they are within the asymptotic limit almost definitely. 
This process of stiffening property is due in part to the law of large num2 bers. The speaker Hachem has been proposed for first deterministic equivalents. In[ 23], Who also demonstrated their capture capability essential measures of system performance. Once applied to finite $\mathrm{M}$ and $\mathrm{K}$ the deterministic equivalents are pionted to as huge scale extrapolations.

A) Imperfect Channel State Information at BSs:

The uplink pilot transmission is used to receive instant CSI based on the TDD protocol of each BS. Each of the UTS in such cells, for the incomplete coherence of the channel of the fading channel, detecting an alternative reciprocal pilot pattern, a set of equal orthogonal chain assemblies is reused. The pilot interference generated from adjacent cells weakens the estimates of the channels [27]. When you test the Terminal K channel of the user in cell $\mathrm{j}$, the rear BS obtains its pilot signal, which is compared to the pilot sequence of this UT.

$$
y_{j, k}^{t r} \approx h_{j, k}+\sum h_{j, l, k}+\frac{1}{\sqrt{\rho}} q_{j, k}^{t r}
$$

where $q_{j, k}^{t r} \sim C N\left(0_{M \times 1}, I_{M}\right)$ and $\rho_{t r}>0$ is effective training $\operatorname{SNR}[30]$.The Matched Filter estimate $h_{j, k}+h_{j, l, k}$ is given as [31].

B) Issues of RZF in compexity:

Where the precoding of the SINRs achieved by RZF converges in the major reign. However, random quantities of precoding matrices which need to be retrieved at the same rate as channel command are modified, so with the typical consistency of a less milliseconds, they are essential to reverse hundreds of times per second to calculate the large-dimensional matrix. The amount of arithmetic activities required for matrix expression grows cubically in the matrix range, rendering this matrix operation inflexible in huge-scale devices, reducing the complexity of implementation and retaining the majority of RZF performance; Precoding of low-complexity TPEs for single-cell systems was proposed in [28] and [29].

The latest precoding strategy has two advantages over RZF pre-coding 1) at the beginning of each coherence interval the pre-coding matrix is not precomputed, so there are no mathematical loops and the mathematical processes are distributed over time uniformly. 2)The precoding method as classified into easy matrix-vector integer arithmetic which can be extremely parallel and implemented.

\section{TPE Precoding}

The idea of truncated polynomial expansion is furnish the scenario with a new type of low complication linear precoding strategy. We recalled that the definition of TPE comes from those in the Cayley-Hamilton theorem stating that it is imaginable to write extreme of a dimension $\mathrm{M}$ matrix $\mathrm{F}$ as a calibrated 
amount of the first M power.

$$
F^{-1}=\frac{(-1)^{M-1}}{\operatorname{det}(F)} \sum_{i=0}^{M-1} \alpha_{l} F^{l}
$$

where $\alpha_{l}$ is the coefficient of the habitual polynomial. The simplified precoding is evaluated by taking only a truncated number of the matrix capabilities

whereas $Z_{j}=0_{M \times M}$ and truncated sequence of TPE precoding $J_{j}$.

$$
\begin{aligned}
U_{j}^{T P E} & =\sum_{n=0}^{J_{j}-1} w_{n, j}\left(\frac{\widehat{H}_{j, j} \widehat{H}_{j, j}^{H}}{K}\right)^{n} \frac{\widehat{H}_{j, j}}{\sqrt{K}} \\
& =\sum_{n=0}^{J_{j}-1} w_{n, j} Q_{n, j} \frac{\widehat{H}_{j, j}}{\sqrt{K}}
\end{aligned}
$$

where

$$
Q_{n, j}=\left(\frac{\widehat{H}_{j, j} \widehat{H}_{j, j}^{H}}{K}\right)^{n}
$$

and $w_{n, j}, j=0, \ldots . J_{j}-1$ are the $J_{j}$ The vector values used in cell $\mathrm{j}$,Meanwhile, only the design parameter is precoded by RZF $\varphi_{J}$, However, the proposed TPE precoding method is a broader set of parameters that $J_{j}$ design. Polynomial coefficients have been used to describe a parameterized class of precoding schemes that range from MRT to RZF precoding when $J_{j}=\min (\mathrm{M}, \mathrm{K})$ and $w_{n, j}$ specific by putting coefficients on the characteristic polynomial of $\sqrt{K}\left(\hat{H} i, j \hat{H} j, j+K \Phi_{j} I_{M}\right)^{-1}$.we mention to $J_{j}$ as the TPE order to the jth cell and the polynomial coefficient in $(28)$ is $J_{j}-1$.For an $J_{j}$ i $\min (\mathrm{M}, \mathrm{K})$, the polynomial coefficients has to use as design variable that would be choose to maximize proper device performance metric [28].An starting option is

$$
w_{n, j}^{\text {initial }}=\beta_{j} k_{j} \sum_{m=n}^{J_{j}-1}\left(\begin{array}{c}
m \\
n
\end{array}\right)\left(1-k_{j} \varphi_{j}\right)^{m-n}\left(-k_{j}\right)^{n}
$$

where $\beta_{j}$ and $\varphi_{j}$ are in RZF precoding,the $k_{j}$ can take any value to $\| I_{M}-$ $k_{j}\left(\frac{1}{K} \widehat{H} \widehat{H}^{H}+\varphi_{j} I_{M}\right)<1$ Calculating the Taylor expansion of the matrix inverse yields this equation. The coefficients in (31) provide performace near to that of ZRF precoding when $J_{j} \rightarrow \infty[28]$.However, we can acquire far and away superior execution than the imperfect RZF, using just little TPE orders 


\section{8 simulation Results}

There are six cases that represent the performance analysis for linear precoding techniques i.e. ZF and MRT, based on the method of vector normalisation, the method of matrix normalisation and the contrast of the two methods of normalization. The findings are presented in displayed achievable sum rate (bit/s/Hz) vs the number of base station antennas and the achievable sum rate vs the number of users.

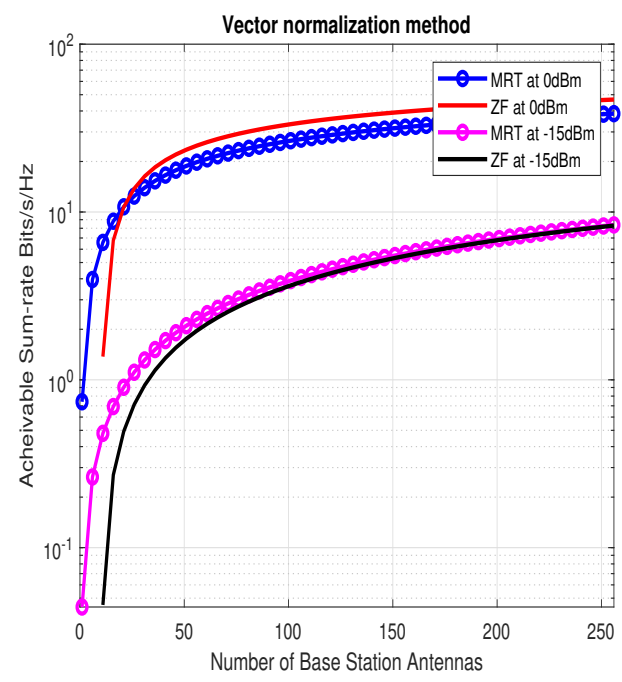

Fig. $3 \mathrm{ZF}$ and MRT efficiency with vector normalization at $\mathrm{K}=10$ users

Figure 3 displays the achievable sum rate according to calculations over the whole antenna spectrum (15 and 18). This metoh is made up of the number of antennas and users $M=1: 256, K=10$. The results show that MRT offers the well act at low power value and better performance when the number of base station antennas at high power is less than 50. On the other hand, ZF provides better high-power efficiency when the number of base station antennas reaches 50

Figure 4 displays the achievable sum rate according to equations over the entire user range (15 and 18). This method made up of the number of antennas and users $\mathrm{M}=256, \mathrm{~K}=1: 10$. The results show that when the number of users up to six users and up to four at high power, MRT delivers the better performance at low power value,ZF gives better results when the number of users at high power is greater than four and at low power is greater than six.

This segment shows the performance of ZF and MRT in single-cell downlink mMIMO network over perfect channel by taking into account the achievable sum rate depending on the method of matrix normalization. Select user num- 


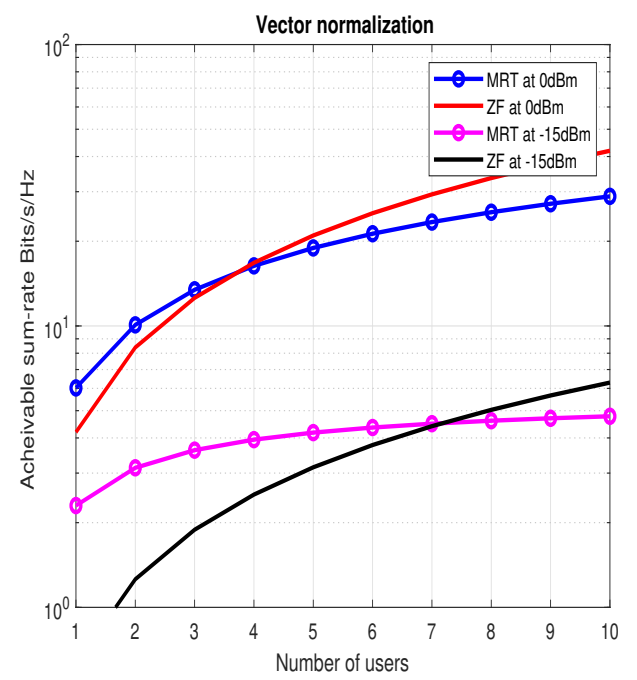

Fig. $4 \mathrm{ZF}$ and MRT efficiency with vector normalization at $\mathrm{M}=256$ and $\mathrm{k}=1: 10$

bers $\mathrm{K}=10$ and antenna numbers are 256. Then set the transmitting power downlink to $0 \mathrm{dBm}$ and $-15 \mathrm{dBm}$ for the BS.

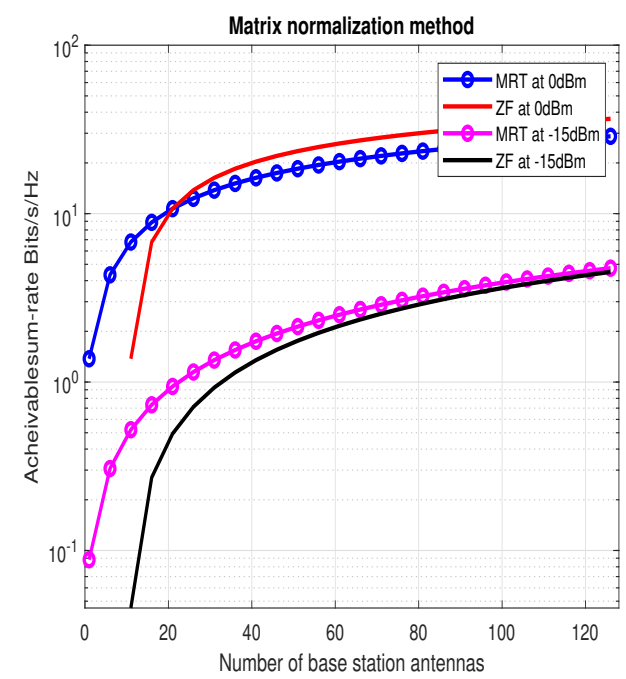

Fig. $5 \mathrm{ZF}$ and MRT efficiency with matrix normalization at $\mathrm{k}=10$ and $\mathrm{M}=1: 256$

Figure 5 shows the achievable sum rate according to the equations over the entire user range (15 and 18). This method is made up of the number of 
antennas and users $\mathrm{M}=1: 256, \mathrm{~K}=10$. The results show that the number of antennas is greater than 22 antennas,ZF fares better at high power value.

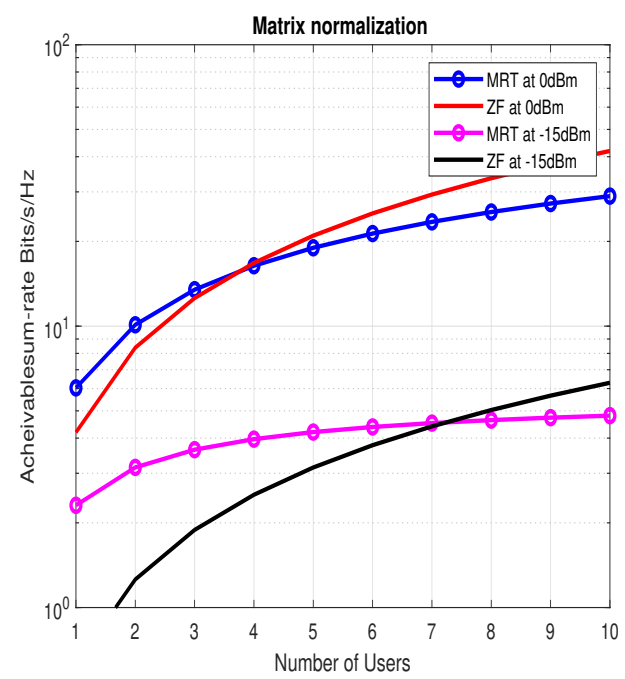

Fig. $6 \mathrm{ZF}$ and MRT efficiency with vector normalization at $\mathrm{k}=10$ and $\mathrm{M}=256$

Figure 6 displays the achievable sum rate according to equations over the ntire user range (15and 18). This method is made up of the number of antennas and users $M=256, K=1: 10$. The results show that when the number of users is less than seven users and less than five users at MRT e at low power value. On the other hand, ZF provides better results when the number of users at high power is greater than four, and at low power is greater than seven.

Figure 7 shows the achievable sum rate according to equations over the entire antenna range ( 15 for $\mathrm{ZF}$ and 18 for MRT). This method is made up of the number of antennas and users $\mathrm{M}=1: 256, \mathrm{~K}=10$. The downlink power transmitted-15dBm. The results show the same output provided by vector and matrix normalization for ZF. The MRT and ZF give the equal performance then the number of antennas greater than 60 at low power value.

This segment gives a numerical approval of the proposed TPE precoding in Figure 8 a down to earth arrangement situation. We consider a four part site $\mathrm{L}=4$ made out of cells and BSs. see Fig.1. Like the channel model introduced in[32], we expect that the UTs in every cell are separated into $\mathrm{G}=$ 2 gatherings in figure 9. UTs of a gathering share roughly a similar area and factual properties

Let's see Fig first. 8. It sees a J=4 TPE order and three separate CSI quality levels at the BS: From Fig. 8, We see it when a poor channel estimate is available, RZF and TPE accomplish nearly the same typical UT performance Additionally, at low SNR values, TPE and RZF perform almost similarly for 


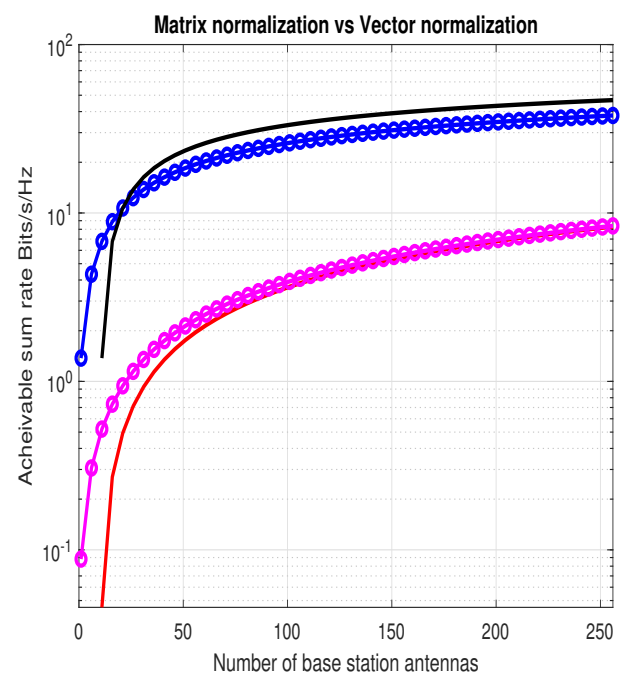

Fig. 7 Matrix versus Vector normalizations at $\mathrm{k}=10, \mathrm{M}=1: 256$ and $\mathrm{Pd}=0 \mathrm{dBm}$

any $\tau$. Generally speaking, the understandable observation is that the difference in the rate increases at high SNRs and when $\tau$ is small.

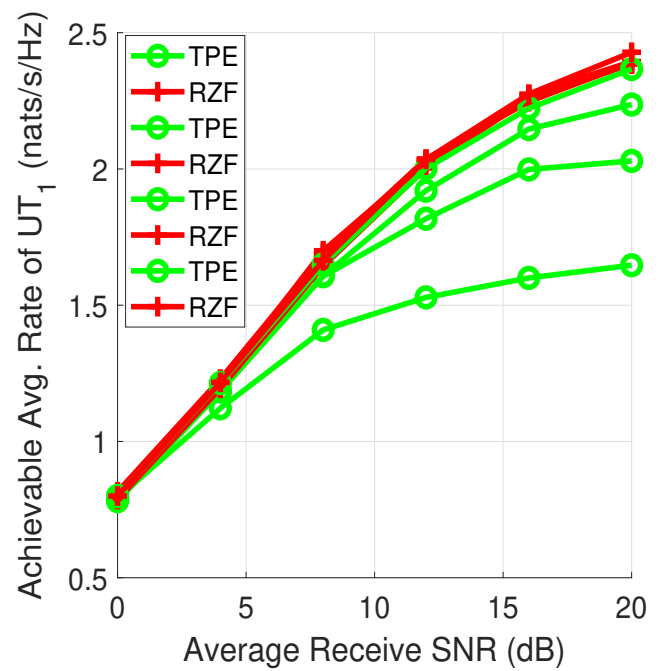

Fig. 8 Average per UT rate vs.SNR ratio for varying CSI errors at the $\mathrm{BS}(\mathrm{J}=5, \mathrm{M}=$ $256, \mathrm{~K}=32)$.

Figure 9 shows the link between the average attainable UT level and TPE order $\mathrm{J}$ in further detail. We consider the scenario $\tau=0.3, \mathrm{M}=512$, and $\mathrm{K}=256$ 
to be in a regime where TPE performs quite poorly (see Fig.8) and precoding complexity becomes an issue. We can observe from the graph that increasing $\mathrm{J}$ to a higher value brings TPE performance closer to RZF's. TPE precoding never outperforms RZF efficiency, which is noteworthy given that TPE has J degrees of freedom to optimize versus RZF's single design parameter.

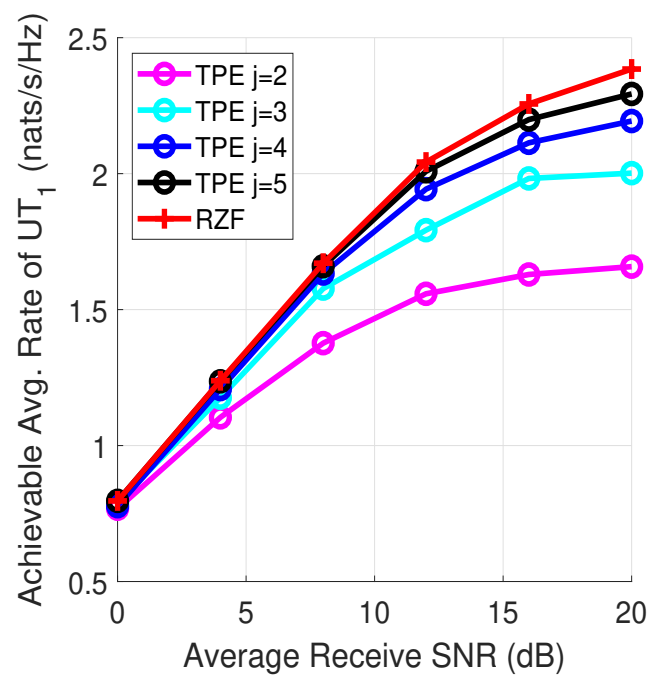

Fig. 9 Rate varies by order. Average UT vs. SNR ratio in the TPE precoding for different orders $\mathrm{J}, \mathrm{M}=512, \mathrm{~K}=256, \tau=0.3)$.

\section{Conclusion}

This paper offers an examination and investigation of direct precoding in mMIMO in single-cell downlink. The parameters contemplated are the achieveable sum rate with a distinction in the quantity of dynamic user and the signal to noise ratio.Simulation results show a superior rate of error created by the MRT precoding scheme. The ZF precoding strategy, in the mean time, gives a superior achievable sum rate.A massive MIMO organize offers the chance to increment achievable sum rate.The achievable sum rate changes for $\mathrm{ZF}$ and MRT when numbering the base station antenna 512.The achievable sum rate upgrades for ZF and MRT (18.8207 dBm and $16.6465 \mathrm{dBm}$ respectively at $0 \mathrm{dBm}$ and $10.2418 \mathrm{dBm}$ and $10.4415 \mathrm{dBm}$ at $15 \mathrm{dBm}$ ); Therefore vector matrix normalization for ZF gives better performance at high downlink transmission power, while above normalizations MRT provides better performance at low downlink transmission power.

This paper sums up the recently proposed TPE precoder to MIMO systems with multicell huge scale. This type of precoders arises from the exceptionally 
mind-complex RZF precoding system through a truncated polynomial expansion that approximates the regularized channel reversal. The model contains basic multi-cell highlights, for example, client explicit channel measurements, different TPE arranges in various cells, and force requirements explicit to the cells. We acquired SINR expressions asymptotic.

\section{Declarations}

\section{Funding}

The authors received no financial support for the research work.

\section{Conflict of interest}

The authors declare that they have no conflict of interest.

\section{Availability of data and material}

Data sharing not applicable to this article as no datasets were generated during the current study.

\section{Code Availability}

Software Application.

\section{References}

[1] .Subuh Pramono, Eddy Triyono on "Comparative Performance Analysis of Linear Precoding in Downlink Multi-user MIMO", Proceeding of EECSI 2018, Malang - Indonesia, 16-18 Oct 2018

[2]. J.C. Guey, P. K. Liao, Y.S. Chen, A. Hsu, C. H. Hwang, G. Lin, "On 5G radio access architecture and technology [Industry Perspectives]," IEEE Wireless Communications, vol. 22, no. 5, October 2015

[3].L. Zhao, K. Zheng, H. L. H. Zhao, W. Wang, "Performance Analysis for Downlink Massive MIMO System with ZF precoding," Transactions on Emerging Telecommunications Technologies, vol.8, no.3, pp. 390-398, 2014.

[4]. H. Quoc Ngo, "Massive MIMO: Fundamentals and System Designs," Linköping University, Sweden, 2015

[5].Nusrat Fatema, Guang Hua, Yong Xiang, Dezhong Peng and Iynkaran Natgunanathan, on "Massive MIMO Linear Precoding: A Survey", IEEE SYSTEMS JOURNAL, VOL. 12, NO. 4, DECEMBER 2018 
[6]. J. Hoydis, S. ten Brink, and M. Debbah, "Massive MIMO in the UL/DL of cellular networks: How many antennas do we need?," vol. 31, no. 2, pp. 160-171, Feb. 2013.

[7]. E. Bjornson, M. Bengtsson, and B. Ottersten, "Pareto characterization of the multicell MIMO performance region with simple receivers," vol. 60, no. 8, pp. 4464-4469, Aug. 2012.

[8] C. B. Peel, B. M. Hochwald, and A. L. Swindlehurst, "A vector perturbation technique for near-capacity multi antenna multiuser communication, Part I: Channel inversion and regularization," vol. 53, no. 1, pp. 195-202, Jan. 2005.

[9] T.K.Y. Lo, "Maximum ratio transmission," vol. 47, no. 10, pp. 14581461, Oct. 1999.

[]10].Y. Zhang, J. Gao, Y. Liu, "MRT precoding in downlink multi-user MIMO systems," EURASIP Journal on Wireless Communications, vol. 241, October 2016.

[11] C. D. Ho, H. Q. Ngo, M. Matthaiou, T.Q. Duong, "On the Performance of Zero-Forcing Processing in Multi-Way Massive MIMO Relay Networks," IEEE Communications Letters, vol. 21, no. 4, pp. 849 - 852, 2017

[12]. T. Parfait, Y. Kuang, andK. Jerry, "Performance analysis and comparison of ZF and MRT based downlink massive MIMO systems," in Proc. 6th Int. Conf. Ubiquitous Future Netw., Shanghai, China, 2014, pp. 383-388.

[13].A.Muller, A.Kammounz, E.Bjornson, and M.Debbahx, "Efficient linear recoding for massive MIMO systems using truncated polynomial expansion," in Proc. 8th Sens. Array Multi channel Signal Process. Workshop, A Coru na, Spain, 2014, pp. 273-276.

[14]. A. Kammoun, A. Muller, E. Bjornson, and M. Debbah, "Linear precoding based on polynomial expansion: Large-scale multi-cell MIMO systems," IEEE J. Sel. Topics Signal Process., vol. 8, no. 5, pp. 861-875, Oct. 2014.

[15]. A. Muller, R. Couillet, E. Bjornson, S. Wagner, and M. Debbah, "Interference-aware RZF precoding for multicell downlink systems," IEEE Trans. Signal Process., vol. 63, no. 15, pp. 3959-3973, Aug. 2015.

[16]. S. Wagner, R. Couillet, M. Debbah, and D. T. M. Slock, "Large system analys is of linear precoding in correlated MISO broadcast channels under limited feedback,"IEEETrans.Inf.Theory, vol.58,no.7,pp.4509-4537, Mar. 2012.

[17] AxelMueller, AblaKammoun, EmilBjörnson "Linear precoding based on polynomial expansion: reducing complexity in massive MIMO",EURASIP Journalon Wireless Communications and Networking (2016) 2016:63, DOI10.1186/s13638$016-0546-z$

[18] Ankita Sahu, Manish Panchal, and Rekha Jain," Energy-Efficient Optimum Design for Massive MIMO", Springer Nature Singapore Pte Ltd. 2018 S. Bhattacharyya et al. (eds.), Advanced Computational and Communication Paradigms, Lecture Notes in Electrical Engineering 475, https://doi.org/10.1007/978981-10-8240-5

[19] M. Joham, W. Utschick, and J. A. Nossek, "Linear transmit processing in MIMO communications systems," IEEE Trans. Signal Process., vol. 53, no. 8, pp. 2700-2712, Aug. 2005. 
[20] A. H. Mehana and A. Nosratinia, "Diversity of MIMO linear precoding," IEEE Trans. Inf. Theory, vol. 60, no. 2, pp. 1019-1038, Nov. 2013

[21].S. Zarei, W. Gerstacker, R. R. Muller, and R. Schober, "Low-Complexity Linear Precoding for Downlink Large-Scale MIMO Systems," in Proc. IEEE PIMRC, 2013

[22] S. Wagner, R. Couillet, M. Debbah, and D. T. M. Slock, "Large System Analysis of Linear Precoding in MISO Broadcast Channels with Limited Feedback," IEEETrans.Inf.Theory, vol.58,no.7,pp.4509-4537,2012

[23]. WHachem ,OKhorunzhy ,PLoubaton ,JNajim ,LAPastur ,Anew approach for capacity analysis of large dimensional multi-antenna channels. IEEETrans.Inf.Theory.54(9),3987-4004(2008)

[24]. VKNguyen, JSEvans,in Global Telecommunications Conference,2008.IEEE GLOBECOM2008. IEEE. Multiuser Transmit Beamforming via Regularized Channel Inversion: A Large System Analysis(IEEE,NewOrleans,LO,2008). doi:10.1109/GLOCOM.2008.ECP.176

[25]. SWagner, RCouillet, MDebbah, DTMSlock, Large system analysis of linear precoding in MIS Obroad cast channels with limited feedback. IEEE Trans.Inf.Theory.58(7),4509-4537(2012)

[26]. RMuharar, JEvans, in Communications (ICC), 2011IEEEInternational Conference on 5-9 June 2011.Downlink Beamforming with Transmit-Side Channel Correlation: A Large System Analysis(IEEE,Kyoto,2011),pp.1-5. doi:10.1109

[27] T.L.Marzetta, "Non cooperative cellular wireless with unlimited numbers of base station antennas," IEEE Trans. Communications., vol. 9, no. 11, pp. 3590-3600, Nov. 2010.

[28] A. Müller, A. Kammoun, E. Björnson, and M. Debbah, "Linear precoding based on polynomial expansion: Reducing complexity in massive MIMO (extended version)," IEEE Trans. Signal Process., Sep. 2013, submitted to arXiv: 1310.1806, submitted for publication.

[29] S. Zarei, W. Gerstacker, R. R. Müller, and R. Schober, "Low-complexity linear precoding for downlink large-scale MIMO systems," in Proc. IEEE Int. Symp. Personal, Indoor, Mobile Radio Commun. (PIMRC), 2013, pp. 1119-1124.

[30] J.Hoydis,S.tenBrink,andM.Debbah, "Massive MIMO in the UL/DL of cellular networks: How many antennas do we need?," IEEE J. Sel. Areas Commun., vol. 31, no. 2, pp. 160-171, Feb. 2013.

[31] E. Björnson and B. Ottersten, "A framework for training-based estimation in arbitrarily correlated rician MIMO channels with Rician disturbance,"IEEE Trans.Signal Process.,vol.58,no.3,pp. 1807-1820, Mar. 2010

[32]. A. Adhikary, J. Nam, J. Y. Ahn, and G. Caire, "Joint spatial division andmultiplexing," [Online]. http://arxiv.org/abs/1209.1402 